Iz-iddine EL-Fassi, Choonkil Park, and Gwang Hui Kim*

\title{
Stability and hyperstability of a quadratic functional equation and a characterization of inner product spaces
}

https://doi.org/10.1515/dema-2018-0027

Received June 14, 2018; accepted October 31, 2018

Abstract: We have proved the Hyers-Ulam stability and the hyperstability of the quadratic functional equation

$$
f(x+y+z)+f(x+y-z)+f(x-y+z)+f(-x+y+z)=4[f(x)+f(y)+f(z)]
$$

in the class of functions from an abelian group $G$ into a Banach space.

Keywords: Hyers-Ulam stability, hyperstability, quadratic functional equation, fixed point theorem

MS: 39B82, 39B52, 47H14, 47H10

\section{Introduction}

In 1940, Ulam [1] gave a wide ranging talk before the mathematics club of the University of Wisconsin in which he discussed a number of important unsolved problems. Among those was the question concerning the stability of homomorphisms:

Let $(G, \cdot)$ be a group and let $\left(G^{\prime}, \cdot \cdot d\right)$ be a metric group with the metric $d$. Given $\epsilon>0$, does there exist $\delta>0$ such that if a mapping $h: G \rightarrow G^{\prime}$ satisfies the inequality

$$
d(h(x \cdot y), h(x) \cdot h(y)) \leq \delta
$$

for all $x, y \in G$, then there is a homomorphism $H: G \rightarrow G^{\prime}$ with

$$
d(h(x), H(x)) \leq \epsilon
$$

for all $x \in G$ ?

Ulam’s problem was partially solved by Hyers [2] in 1941.

Theorem 1. [2] Let $E$ be a normed vector space, $F$ a Banach space and suppose that the mapping $f: E \rightarrow F$ satisfies the inequality

$$
\|f(x+y)-f(x)-f(y)\| \leq \epsilon
$$

for all $x, y \in E$, where $\epsilon$ is a constant. Then the limit

$$
T(x)=\lim _{n \rightarrow \infty} 2^{-n} f\left(2^{n} x\right)
$$

Iz-iddine EL-Fassi: Department of Mathematics, Faculty of Sciences, University of Ibn Tofail, Kenitra, Morocco; E-mail: izidd-math@hotmail.fr

Choonkil Park: Research Institute for Natural Sciences, Hanyang University, Seoul 04763, Republic of Korea; E-mail: baak@hanyang.ac.kr

${ }^{\star}$ Corresponding Author: Gwang Hui Kim: Department of Mathematics, Kangnam University, Yongin, Gyoenggi 16979,

Republic of Korea; E-mail: ghkim@kangnam.ac.kr 
exists for each $x \in E$ and $T$ is the unique additive mapping satisfying

$$
\|f(x)-T(x)\| \leq \epsilon
$$

for all $x \in E$. Also, if for each $x$ the function $t \rightarrow f(t x)$ from $\mathbb{R}$ to $F$ is continuous for each fixed $x$, then $T$ is linear. If $f$ is continuous at a single point of $E$, then $T$ is continuous in $E$.

Bourgin [3], Aoki [4], Rassias [5] and Gajda [6] treated this problem for approximate additive mappings controlled by unbounded functions.

Theorem 2. Let $f: E \rightarrow F$ be a mapping from a real normed vector space $E$ into a Banach space $F$ satisfying the inequality

$$
\|f(x+y)-f(x)-f(y)\| \leq \theta\left(\|x\|^{p}+\|y\|^{p}\right)
$$

for all $x, y \in E \backslash\{0\}$, where $\theta$ and $p$ are constants with $\theta>0$ and $p \neq 1$. Then there exists $a$ unique additive mapping $T: E \rightarrow F$ such that

$$
\|f(x)-T(x)\| \leq \frac{\theta}{\left|1-2^{p-1}\right|}\|x\|^{p}
$$

for all $x \in E \backslash\{0\}$.

Theorem 2 is due to Aoki [4] for $0<p<1$ (see also [5]); Gajda [6] for $p>1$; Hyers [2] for $p=0$ and Rassias [7] for $p<0$ (see [3, 8]).

In 1994, Găvruta [9] generalized these theorems for approximate additive mappings controlled by the unbounded Cauchy difference with regular conditions, i.e., he replaced $\theta\left(\|x\|^{p}+\|y\|^{p}\right)$ with a general control function $\varphi(x, y)$.

The stability problems of several functional equations have been extensively investigated by a number of authors and there are many interesting results concerning this problem (see [10-13]).

Recently, interesting results concerning quadratic functional equation

$$
f(x+y+z)+f(x+y-z)+f(x-y+z)+f(-x+y+z)=4[f(x)+f(y)+f(z)]
$$

have been obtained in $[14,15]$.

Lemma 1. [14] Let $X$ and $Y$ be vector spaces over fields of characteristic different from 2, respectively. A mapping $f: X \rightarrow Y$ satisfies (1.3) if and only if the mapping $f: X \rightarrow Y$ is a solution of the quadratic equation $f(x+y)+$ $f(x-y)=2 f(x)+2 f(y)$.

We say a functional equation $\mathfrak{D}$ is hyperstable if any function $f$ satisfying the equation $\mathfrak{D}$ is approximately a true solution of $\mathfrak{D}$. The term hyperstability was used for the first time probably in [16]. However, it seems that the first hyperstability result was published in [3] and concerned the ring homomorphisms. The hyperstability results for Cauchy equation were investigated by Brzdęk [17-19]. Gselmann [20] studied the hyperstability of the parametric fundamental equation of information. In [21], Bahyrycz and Piszczek provided the hyperstability of the Jensen functional equation. For more information on hyperstability of functional equations, see [22].

Throughout this paper, we will denote the set of natural numbers by $\mathbb{N}$, the set of integers by $\mathbb{Z}$ and the set of real numbers by $\mathbb{R}$. Let $\mathbb{N}^{*}$ be the set of positive integers. We denote that $\mathbb{N}_{m_{0}}$ (with $m_{0} \in \mathbb{N}^{*}$ ) the set of all integers greater than or equal to $m_{0}$. Let $\mathbb{R}_{+}:=[0, \infty)$ be the set of nonnegative real numbers and $Y^{X}$ denote the family of all functions mapping from a nonempty set $X$ into a nonempty set $Y$.

In this paper, we present the stability and hyperstability results for the quadratic functional equation (1.3) in the class of functions from a commutative group $(G,+)$ into a Banach space $E$.

The method of the proof of the main results is motivated by an idea used in [17-19, 23, 24]. It is based on a fixed point theorem for functional spaces obtained by Brzdȩk et al. (see [25, Theorem 1]). 
First, we take the following three hypotheses (all notations come from [25]):

(H1) $U$ is a nonempty set, $V$ is a Banach space, $f_{1}, \ldots . f_{k}: U \rightarrow U$ and $L_{1}, \ldots . L_{k}: U \rightarrow \mathbb{R}_{+}$are given.

(H2) $\mathcal{T}: V^{U} \rightarrow V^{U}$ is an operator satisfying the inequality

$$
\|\mathcal{T} \xi(x)-\mathcal{T} \mu(x)\| \leq \sum_{i=1}^{k} L_{i}(x)\left\|\xi\left(f_{i}(x)\right)-\mu\left(f_{i}(x)\right)\right\|
$$

for all $\xi, \mu \in V^{U}, x \in U$.

(H3) $\Lambda: \mathbb{R}_{+}^{U} \rightarrow \mathbb{R}_{+}^{U}$ is a linear operator defined by

$$
\Lambda \delta(x):=\sum_{i=1}^{k} L_{i}(x) \delta\left(f_{i}(x)\right)
$$

for all $\delta \in \mathbb{R}_{+}^{U}, x \in U$.

The mentioned fixed point theorem is stated as follows.

Theorem 3. Let (H1)-(H3) be valid and functions $\varepsilon: U \rightarrow \mathbb{R}_{+}$and let $\varphi: U \rightarrow V$ fulfil the following two conditions:

$$
\begin{gathered}
\|\mathcal{T} \varphi(x)-\varphi(x)\| \leq \varepsilon(x), \quad x \in U, \\
\varepsilon^{\star}(x):=\sum_{n=0}^{\infty} \Lambda^{n} \varepsilon(x)<\infty, \quad x \in U .
\end{gathered}
$$

Then there exists a unique fixed point $\psi$ of $\mathcal{T}$ with

$$
\|\varphi(x)-\psi(x)\| \leq \varepsilon^{\star}(x), \quad x \in U .
$$

Moreover

$$
\psi(x)=\lim _{n \rightarrow \infty} \mathcal{T}^{n} \varphi(x), \quad x \in U
$$

\section{Main results}

The following theorems are the main results in this paper and concern the stability of the functional equation (1.3).

Theorem 4. Let $(G,+)$ be an abelian group and $E$ be a Banach space. Let $f: G \rightarrow E, \varphi: G^{3} \rightarrow[0, \infty)$ and $u: \mathbb{Z}^{*}=\mathbb{Z} \backslash\{0\} \rightarrow[0, \infty)$ be functions satisfying the following three conditions

$$
\begin{gathered}
\mathcal{M}:=\left\{m \in \mathbb{Z}^{\star}: 2 u(2 m+1)+8 u(-m)+u(-4 m-1)<1\right\} \neq \emptyset, \\
\varphi(t x, t y, t z) \leq u(t) \varphi(x, y, z)
\end{gathered}
$$

and

$$
\|f(x+y+z)+f(x+y-z)+f(x-y+z)+f(-x+y+z)-4[f(x)+f(y)+f(z)]\| \leq \varphi(x, y, z)
$$

for all $x, y, z \in G, t \in\{2 m+1,-m,-4 m-1\}$ and $m \in \mathcal{M}$. Then there exists a unique mapping $Q: G \rightarrow E$ satisfying (1.3) and

$$
\|f(x)-Q(x)\| \leq \phi(x)
$$

where

$$
\phi(x):=\inf \left\{\frac{\varphi((2 m+1) x,-m x,-m x)}{1-2 u(2 m+1)-8 u(-m)-u(-4 m-1)}: m \in \mathcal{M}\right\}
$$

for all $x \in G$. 
Proof. Replacing $(x, y, z)$ by $((2 m+1) x,-m x,-m x)$ in $(2.3)$, we get

$$
\|2 f((2 m+1) x)+8 f(-m x)-f((-4 m-1) x)-f(x)\| \leq \varphi((2 m+1) x,-m x,-m x):=\varepsilon_{m}(x)
$$

for all $x \in G$ and $m \in \mathbb{Z}^{\star}$. Further put

$$
\mathcal{T} \xi(x):=2 \xi((2 m+1) x)+8 \xi(-m x)-\xi((-4 m-1) x), \quad x \in G, \xi \in E^{G}, m \in \mathbb{Z}^{\star} .
$$

Then the inequality (2.5) takes the form

$$
\|\mathcal{T} f(x)-f(x)\| \leq \varepsilon_{m}(x), \quad x \in G .
$$

Now, we define an operator $\Lambda: \mathbb{R}_{+}^{G} \rightarrow \mathbb{R}_{+}^{G}$ for $m \in \mathbb{Z}^{*}$ by

$$
\Lambda \delta(x):=2 \delta((2 m+1) x)+8 \delta(-m x)+\delta((-4 m-1) x), x \in G, \quad \delta \in \mathbb{R}_{+}^{G} .
$$

This operator has the form described in (H3) with $k=4$ and $f_{1}(x)=(2 m+1) x, f_{2}(x)=-m x, f_{3}(x)=(-4 m-1) x$, $L_{1}(x)=2, L_{2}(x)=8$ and $L_{3}(x)=1$ for $x \in G$.

Moreover, for every $\xi, \mu \in E^{G}$ and $x \in G$, we obtain

$$
\begin{aligned}
\|\mathcal{T} \xi(x)-\mathcal{T} \mu(x)\| & =\left\|2(\xi-\mu)\left(f_{1}(x)\right)+8(\xi-\mu)\left(f_{2}(x)\right)-(\xi-\mu)\left(f_{3}(x)\right)\right\| \\
& \leq 2\left\|(\xi-\mu)\left(f_{1}(x)\right)\right\|+8\left\|(\xi-\mu)\left(f_{2}(x)\right)\right\|+\left\|(\xi-\mu)\left(f_{3}(x)\right)\right\| \\
& =\sum_{i=1}^{4} L_{i}(x)\left\|(\xi-\mu)\left(f_{i}(x)\right)\right\|,
\end{aligned}
$$

where $(\xi-\mu)(y)=\xi(y)-\mu(y)$ for all $y \in G$. So (H2) is valid. It is easy to check that

$$
\begin{aligned}
\Lambda \varepsilon_{k}(x) & =2 \varepsilon_{k}((2 m+1) x)+8 \varepsilon_{k}(-m x)+\varepsilon_{k}((-4 m-1) x) \\
& \leq 2 u(2 m+1) \varepsilon_{k}(x)+8 u(-m) \varepsilon_{k}(x)+u(-4 m-1) \varepsilon_{k}(x) \\
& =[2 u(2 m+1)+8 u(-m)+u(-4 m-1)] \varepsilon_{k}(x)
\end{aligned}
$$

for all $x \in G, k \in \mathbb{Z}^{\star}$ and $m \in \mathcal{M}$. Therefore, since the operator $\Lambda$ is linear, we have

$$
\begin{aligned}
\varepsilon^{\star}(x): & =\sum_{n=0}^{\infty} \Lambda^{n} \varepsilon_{m}(x) \\
& \leq \sum_{n=0}^{\infty}(2 u(2 m+1)+8 u(-m)+u(-4 m-1))^{n} \varepsilon_{m}(x) \\
& =\frac{\varepsilon_{m}(x)}{1-2 u(2 m+1)-8 u(-m)-u(-4 m-1)}<\infty
\end{aligned}
$$

for all $x \in G$ and $m \in \mathcal{M}$. Thus, according to Theorem 3, for each $m \in \mathcal{M}$ there exists a unique mapping $Q_{m}: G \rightarrow E$ such that

$$
\begin{gathered}
Q_{m}(x)=2 Q_{m}((2 m+1) x)+8 Q_{m}(-m x)-Q_{m}((-4 m-1) x), \quad x \in G \\
\left\|f(x)-Q_{m}(x)\right\| \leq \frac{\varepsilon_{m}(x)}{1-2 u(2 m+1)-8 u(-m)-u(-4 m-1)}
\end{gathered}
$$

for all $x \in G$ and $m \in \mathcal{M}$. Moreover

$$
Q_{m}(x)=\lim _{n \rightarrow \infty} \mathcal{T}^{n} f(x), \quad x \in G, \quad m \in \mathcal{M}
$$

Next, we show that

$$
\| \mathcal{T}^{n} f(x+y+z)+\mathcal{T}^{n} f(x+y-z)+\mathcal{T}^{n} f(x-y+z)+\mathcal{T}^{n} f(y-x+z)-4 \mathcal{T}^{n} f(x)
$$




$$
-4 \mathcal{T}^{n} f(y)-4 \mathcal{T}^{n} f(z) \| \leq(2 u(2 m+1)+8 u(-m)+u(-4 m-1))^{n} \varphi(x, y, z)
$$

Fix $m \in \mathcal{M}$. Indeed, if $n=0$, then (2.11) is simply (2.3). So, fix $n \in \mathbb{N}$ and suppose that (2.11) holds for $n$. Then

$$
\begin{aligned}
& \| \mathcal{T}^{n+1} f(x+y+z)+\mathcal{T}^{n+1} f(x+y-z)+\mathcal{T}^{n+1} f(x-y+z)+\mathcal{T}^{n+1} f(y-x+z) \\
& -4 \mathcal{T}^{n+1} f(x)-4 \mathcal{T}^{n+1} f(y)-4 \mathcal{T}^{n+1} f(z) \| \\
& =\| 2 \mathcal{T}^{n} f((2 m+1)(x+y+z))+8 \mathcal{T}^{n} f(-m(x+y+z)) \\
& -\mathcal{T}^{n} f((-4 m-1)(x+y+z))+2 \mathcal{T}^{n} f((2 m+1)(x+y-z)) \\
& +8 \mathcal{T}^{n} f(-m(x+y-z))-\mathcal{T}^{n} f((-4 m-1)(x+y-z)) \\
& +2 \mathcal{T}^{n} f((2 m+1) x-y+z)+8 \mathcal{T}^{n} f(-m(x-y+z)) \\
& -\mathcal{T}^{n} f((-4 m-1) x-y+z)+2 \mathcal{T}^{n} f((2 m+1)(-x+y+z)) \\
& +8 \mathcal{T}^{n} f(-m(-x+y+z))-\mathcal{T}^{n} f((-4 m-1)(-x+y+z)) \\
& -4\left[2 \mathcal{T}^{n} f((2 m+1) x)+8 \mathcal{T}^{n} f(-m x)-\mathcal{T}^{n} f((-4 m-1) x)\right] \\
& -4\left[2 \mathcal{T}^{n} f((2 m+1) y)+8 \mathcal{T}^{n} f(-m y)-\mathcal{T}^{n} f((-4 m-1) y)\right] \\
& -4\left[2 \mathcal{T}^{n} f((2 m+1) z)+8 \mathcal{T}^{n} f(-m z)-\mathcal{T}^{n} f((-4 m-1) z)\right] \| \\
& \leq 2(2 u(2 m+1)+8 u(-m)+u(-4 m-1))^{n} \varphi((2 m+1) x,(2 m+1) y,(2 m+1) z) \\
& +8(2 u(2 m+1)+8 u(-m)+u(-4 m-1))^{n} \varphi(-m x,-m y,-m z) \\
& +(2 u(2 m+1)+8 u(-m)+u(-4 m-1))^{n} \varphi((-4 m-1) x,(-4 m-1) y,(-4 m-1) z) \\
& \leq(2 u(2 m+1)+8 u(-m)+u(-4 m-1))^{n+1} \varphi(x, y, z)
\end{aligned}
$$

for all $x, y, z \in G$.

Thus, by induction, we obtain that (2.11) holds for all $x, y, z \in G$ and for all $n \in \mathbb{N}$. Letting $n \rightarrow \infty$ in (2.11), we obtain that

$$
Q_{m}(x+y+z)+Q_{m}(x+y-z)+Q_{m}(x-y+z)+Q_{m}(-x+y+z)=4\left[Q_{m}(x)+Q_{m}(y)+Q_{m}(z)\right]
$$

for all $x, y, z \in G$ and $m \in \mathcal{M}$ such that

$$
\left\|f(x)-Q_{m}(x)\right\| \leq \frac{\varepsilon_{m}(x)}{1-2 u(2 m+1)-8 u(-m)-u(-4 m-1)} .
$$

Now, we prove that $Q_{m}=Q_{k}$ for all $m, k \in \mathcal{M}$. Let us fix $m, k \in \mathcal{M}$ and note that $Q_{k}$ satisfie (2.9) with $m$ replaced by $k$. Hence, by replacing $(x, y, z)$ by $((2 m+1) x,-m x,-m x)$ in (2.12), we get $\mathcal{T} Q_{j}=Q_{j}$ for $j=m, k$ and

$$
\left\|Q_{m}(x)-Q_{k}(x)\right\| \leq \frac{\varepsilon_{m}(x)}{1-2 u(2 m+1)-8 u(-m)-u(-4 m-1)}+\frac{\varepsilon_{k}(x)}{1-2 u(2 k+1)-8 u(-k)-u(-4 k-1)}
$$

for all $x \in G$. It follows from the linearity of $\Lambda$ and (2.7) that

$$
\begin{aligned}
\| Q_{m}(x) & -Q_{k}(x)\|=\| \mathcal{T}^{n} Q_{m}(x)-\mathcal{T}^{n} Q_{k}(x) \| \\
& \leq \frac{\Lambda^{n} \varepsilon_{m}(x)}{1-2 u(2 m+1)-8 u(-m)-u(-4 m-1)}+\frac{\Lambda^{n} \varepsilon_{k}(x)}{1-2 u(2 k+1)-8 u(-k)-u(-4 k-1)} \\
& \leq(2 u(2 m+1)+8 u(-m)+u(-4 m-1))^{n} A_{m}(x)+(2 u(2 k+1)+8 u(-k)+u(-4 k-1))^{n} A_{k}(x),
\end{aligned}
$$

where

$$
A_{m}(x):=\frac{\varepsilon_{m}(x)}{1-2 u(2 m+1)-8 u(-m)-u(-4 m-1)}
$$

for all $x \in G$ and $n \in \mathbb{N}$. Letting $n \rightarrow \infty$, we get $Q_{m}=Q_{k}=: Q$. Thus we have

$$
\|f(x)-Q(x)\| \leq A_{m}(x), \quad x \in G, \quad m \in \mathcal{M}
$$


and so we obtain (2.4).

In view of 2.12, it is easy to notice that $Q$ is a solution of (1.3).

To prove the uniqueness of the mapping $Q$, let us assume that there exists a mapping $Q^{\prime}: G \rightarrow E$ which satisfies (1.3) and the inequality

$$
\left\|f(x)-Q^{\prime}(x)\right\| \leq \phi(x), \quad x \in G .
$$

Then

$$
\left\|Q(x)-Q^{\prime}(x)\right\| \leq 2 \phi(x), \quad x \in G .
$$

Further $\mathcal{T} Q^{\prime}(x)=Q^{\prime}(x)$ for all $x \in G$. Consequently, with a fixed $m \in \mathcal{M}$

$$
\begin{aligned}
\left\|Q(x)-Q^{\prime}(x)\right\| & =\left\|\mathcal{T}^{n} Q(x)-\mathcal{T}^{n} Q^{\prime}(x)\right\| \\
& \leq 2 \Lambda^{n} \phi(x) \\
& \leq \frac{2 \Lambda^{n} \varepsilon_{m}(x)}{1-2 u(2 m+1)-8 u(-m)-u(-4 m-1)} \\
& \leq \frac{2[2 u(2 m+1)+8 u(-m)+u(-4 m-1)]^{n} \varepsilon_{m}(x)}{1-2 u(2 m+1)-8 u(-m)-u(-4 m-1)}
\end{aligned}
$$

for all $x \in G$ and $n \in \mathbb{N}$. Letting $n \rightarrow \infty$, we get $Q=Q^{\prime}$. The proof of the theorem is complete.

In a similar way, we can prove Theorem 5 if the inequality (2.3) is defined on $G \backslash\{0\}:=G_{0}$.

Theorem 5. Let $(G,+)$ be an abelian group and $E$ be a Banach space. Let $f: G \rightarrow E, \varphi: G_{0}^{3} \rightarrow[0, \infty)$ and $u: \mathbb{Z}^{*}=\mathbb{Z} \backslash\{0\} \rightarrow[0, \infty)$ be functions satisfying the following three conditions

$$
\begin{gathered}
\mathcal{M}:=\left\{m \in \mathbb{Z}^{\star}: 2 u(2 m+1)+8 u(-m)+u(-4 m-1)<1\right\} \neq \emptyset, \\
\varphi(t x, t y, t z) \leq u(t) \varphi(x, y, z)
\end{gathered}
$$

and

$$
\|f(x+y+z)+f(x+y-z)+f(x-y+z)+f(-x+y+z)-4[f(x)+f(y)+f(z)]\| \leq \varphi(x, y, z)
$$

for all $x, y, z \in G_{0}, t \in\{2 m+1,-m,-4 m-1\}$ and $m \in \mathcal{M}$ with $f(0)=0$. Then there exists a unique mapping $Q: G \rightarrow$ E satisfying (1.3) and

$$
\|f(x)-Q(x)\| \leq \phi(x)
$$

where

$$
\phi(x):=\inf \left\{\frac{\varphi((2 m+1) x,-m x,-m x)}{1-2 u(2 m+1)-8 u(-m)-u(-4 m-1)}: m \in \mathcal{M}\right\}
$$

for all $x \in G_{0}$.

Corollary 1. Let $(G,+)$ be a commutative group and $E$ be a Banach space. Let $f: G \rightarrow E, \varphi: G^{3} \rightarrow[0, \infty)$ and $u: \mathbb{Z}^{\star}=\mathbb{Z} \backslash\{0\} \rightarrow[0, \infty)$ be functions and the conditions (2.1), (2.2) and (2.3) be valid. Assume that

$$
\begin{aligned}
& \inf \{\varphi((2 m+1) x,-m x,-m x): m \in \mathcal{M}\}=0, \\
& \lim _{m \rightarrow \infty}(2 u(2 m+1)+8 u(-m)+u(-4 m-1))=0
\end{aligned}
$$

for all $x \in G$. Then $f$ satisfies (1.3) on $G$.

Proof. Suppose that

$$
\inf \{\varphi((2 m+1) x,-m x,-m x): m \in \mathcal{M}\}=0
$$

for all $x \in G$. Hence from Theorem 4 we have $\phi(x)=0$ for all $x \in G$. Then $f$ satisfies (1.3) on $G$. 
Remark 1. In Theorem 4, if

$$
\liminf _{m \rightarrow \infty}(2 u(2 m+1)+8 u(-m)+u(-4 m-1))=0
$$

(this is the case when, e.g., $\lim _{|m| \rightarrow \infty} u(m)=0$ ), then (2.1) holds and

$$
\phi(x)=\inf _{m \in \mathcal{M}} \varphi((2 m+1) x,-m x,-m x)
$$

for all $x \in G$.

Now we give some applications of Theorem 5 to some cases:

$$
\varphi_{1}(x, y, z)=\theta\|x\|^{p} \cdot\|y\|^{q} \cdot\|z\|^{r}, \quad p+q+r<0
$$

and

$$
\varphi_{2}(x, y, z)=\theta\left(\|x\|^{p}+\|y\|^{p}+\|z\|^{p}\right), \quad p<0,
$$

where $\varphi(x, y, z)=\varphi_{j}(x, y, z)$ for $j \in\{1,2\}, \theta \in \mathbb{R}_{+}, p, q, r \in \mathbb{R}$ and $x, y, z \neq 0$.

Corollary 2. Let $E_{1}$ and $E_{2}$ be a normed space and a Banach space, respectively. Assume $S:=(S,+)$ is a subgroup of the group $\left(E_{1},+\right), p, q, r \in \mathbb{R}, p+q+r<0$ and $\theta \geq 0$. If $f(0)=0$ and $f: S \rightarrow E_{2}$ satisfies

$$
\|f(x+y+z)+f(x+y-z)+f(x-y+z)+f(y-x+z)-4[f(x)+f(y)+f(z)]\| \leq \theta\|x\|^{p}\|y\|^{q}\|z\|^{r}
$$

for all $x, y, z \in S \backslash\{0\}$, then $f$ is a solution of (1.3) on $S$.

Proof. Let $\varphi_{1}(x, y, z)=\theta\|x\|^{p} \cdot\|y\|^{q} \cdot\|z\|^{r}$ and $u(t)=|t|^{p+q+r}$ in Theorem 5 where $p, q, r \in \mathbb{R}, p+q+r<0$ and $t \in \mathbb{Z}^{*}$. Then we observe that condition (2.14) is valid. Obviously, (2.17) and (2.18) hold and there exists $m_{0} \in \mathbb{N}^{\star}$ such that

$$
2|2 m+1|^{p+q+r}+8|m|^{p+q+r}+|4 m+1|^{p+q+r}<1, \quad m \geq m_{0} .
$$

So we obtain (2.13), as well. Consequently, by Corollary 1, every mapping $f: S \rightarrow E_{2}$ fulfilling (2.15) satisfies (1.3) on $S$.

Corollary 3. Let $E_{1}$ and $E_{2}$ be a normed space and a Banach space, respectively. Assume $S:=(S,+)$ is a subgroup of the group $\left(E_{1},+\right), p \in \mathbb{R}, p<0$ and $\theta \geq 0$. If $f(0)=0$ and $f: S \rightarrow E_{2}$ satisfies

$$
\|f(x+y+z)+f(x+y-z)+f(x-y+z)+f(y-x+z)-4[f(x)+f(y)+f(z)]\| \leq \theta\left(\|x\|^{p}+\|y\|^{p}+\|z\|^{p}\right)
$$

for all $x, y, z \in S \backslash\{0\}$, then $f$ is a solution of (1.3) on $S$.

Proof. Let $\varphi_{2}(x, y, z)=\theta\left(\|x\|^{p}+\|y\|^{p}+\|z\|^{p}\right)$ and $u(t)=|t|^{p}$ in Theorem 5 where $p \in \mathbb{R}, p<0$ and $t \in \mathbb{Z}^{*}$. Then we observe that condition (2.14) is valid. Obviously, (2.17) and (2.18) hold and there exists $m_{0} \in \mathbb{N}^{*}$ such that

$$
2|2 m+1|^{p}+8|m|^{p}+|4 m+1|^{p}<1, \quad m \geq m_{0} .
$$

So we obtain (2.13), as well. Consequently, by Corollary 1, every mapping $f: S \rightarrow E_{2}$ fulfilling (2.15) satisfies (1.3) on $S$.

We know that any norm that satisfies the parallelogram law is bound to have been originated from a scalar product. The following corollary gives a characterization of the inner product space, which is one of the applications of Corollaries 2 and 3.

Corollary 4. Let $X$ be a normed space and $X_{0}=X \backslash\{0\}$. Write

$$
\Delta(x, y, z)=\left|\|x+y+z\|^{2}+\|x+y-z\|^{2}+\|x-y+z\|^{2}+\|y-x+z\|^{2}-4\left[\|x\|^{2}+\|y\|^{2}+\|z\|^{2}\right]\right|
$$

for all $x, y \in X$. Assume that one of the following two hypotheses is valid 
(i) $\sup _{x, y, z \in X_{0}} \frac{\Delta(x, y, z)}{\|x\|^{p}\|y\|^{q}\|z\|^{r}}<\infty$ for $p+q+r<0$,

(ii) $\sup _{x, y, z \in X_{0}} \frac{\Delta(x, y, z)}{\|x\|^{p}+\|y\|^{p}+\|z\|^{p}}<\infty$ for $p<0$.

Then $X$ is an inner product space.

Proof. Write $f(x)=\|x\|^{2}$. Then from Corollaries 2 and 3, we easily derive $f$ which is a solution of the functional equation (1.3). That implies $\Delta(x, y)=0$. Thus the norm $\|\cdot\|$ on $X$ satisfies the parallelogram low:

$$
\|x+y\|^{2}+\|x-y\|^{2}=2\|x\|^{2}+2\|y\|^{2}, \quad x, y \in X .
$$

Therefore, $X$ is an inner product space.

Corollary 5. Let $G$ be a commutative group and $E$ be a Banach space. Let $\varphi: G^{3} \rightarrow[0, \infty)$ and $u: \mathbb{Z}^{\star}=$ $\mathbb{Z} \backslash\{0\} \rightarrow[0, \infty)$ be functions and the conditions (2.1), (2.2), (2.17) and (2.18) be valid. If $F: G^{3} \rightarrow E$ is a mapping such that $F\left(x_{0}, y_{0}, z_{0}\right) \neq 0$ for some $x_{0}, y_{0}, z_{0} \in G$ and

$$
\|F(x, y, z)\| \leq \varphi(x, y, z)
$$

for all $x, y, z \in G$, then the functional equation

$$
g(x+y+z)+g(x-y+z)+g(y-x+z)+g(x+y-z)=F(x, y, z)+4[g(x)+g(y)+g(z)], \quad x, y, z \in G
$$

has no solution in the class of functions $g: G \rightarrow E$.

Proof. Suppose that $g: G \rightarrow E$ is a solution to (2.19). Then (2.3) holds, and consequently, according to Corollary $1, g$ satisfies (1.3) on $G$, which means that $F\left(x_{0}, y_{0}, z_{0}\right)=0$. This is a contradiction.

\section{Conclusions}

We have proved the Hyers-Ulam stability and the hyperstability of the quadratic functional equation

$$
f(x+y+z)+f(x+y-z)+f(x-y+z)+f(-x+y+z)=4[f(x)+f(y)+f(z)]
$$

in the class of functions from an abelian group $G$ into a Banach space.

Authors contributions: All authors contributed equally to the writing of this paper. All authors read and approved the final manuscript.

Conflicts of Interest: The authors declare that they have no competing interests.

Acknowledgement: The third author of this work was supported by Basic Science Research Program through the National Research Foundation of Korea funded by the Ministry of Education, Science and Technology (2015R1D1A1A01058083).

\section{References}

[1] Ulam S. M., Problems in Modern Mathematics, Chapter IV, Science Editions, Wiley, New York, 1960

[2] Hyers D. H., On the stability of the linear functional equation, Proc. Nat. Acad. Sci. U.S.A., 1941, 27(4), 222-224 
[3] Bourgin D. G., Approximately isometric and multiplicative transformations on continuous function rings, Duke Math. J., 1949, 16(2), 385-397

[4] Aoki T., On the stability of the linear transformation in Banach spaces, J. Math. Soc. Japan, 1950, 2(1-2), 64-66

[5] Rassias T. M., On the stability of the linear mapping in Banach spaces, Proc. Am. Math. Soc., 1978, 72, 297-300

[6] Gajda Z., On stability of additive mappings, Int. J. Math. Math. Sci., 1991, 14(3), 431-434

[7] Rassias T. M., On a modified Hyers-Ulam sequence, J. Math. Anal. Appl., 1991, 158(1), 106-113

[8] Rassias T. M., Semrl P., On the behavior of mappings which do not satisfy Hyers-Ulam stability, Proc. Am. Math. Soc., 1992, 114(4), 989-993

[9] Găvruta P., A generalization of the Hyers-Ulam-Rassias stability of approximately additive mappings, J. Math. Anal, Appl., 1994, 184(3), 431-436

[10] Czerwik S., On the stability of the quadratic mapping in normed spaces, Bull. Abh. Math. Sem. Univ. Hamburg, 1992, 62(1), 59-64

[11] Gordji M. E., Rahimi A., Park C., Shin D., Ternary Jordan bi-homomorphisms in Banach Lie triple systems, J. Comput. Anal. Appl., 2016, 21(6), 1040-1045

[12] Jung S., On the Hyers-Ulam-Rassias stability of the quadratic functional equations, J. Math. Anal. Appl., 1998, 232(2), 384339

[13] Kannappan P., Quadratic functional equation and inner product spaces, Results Math., 1995, 27(3-4), 368-372

[14] Jung S., Quadratic functional equations of Pexider type, Int. J. Math. Math. Sci., 2000, 24(5), 351-359

[15] Krishnan R., Kumar M. A., On the generalized Hyers-Ulam-Rassias stability of a quadratic functional equation, Int. J. Pure Appl. Math., 2006, 28, 85-94

[16] Maksa G., Páles Z., Hyperstability of a class of linear functional equations, Acta Math. Acad. Paedagog. Nyházi, 2001, 17(2), 107-112

[17] Brzdęk J., Remarks on hyperstability of the the Cauchy equation, Aequationes Math., 2013, 86, 255-267

[18] Brzdęk J., Hyperstability of the Cauchy equation on restricted domains, Acta Math. Hungar., 2013, 141(1-2), 58-67

[19] Brzdęk J., A hyperstability result for the Cauchy equation, Bull. Austral. Math. Soc., 2014, 89(1), 33-40

[20] Gselmann E., Hyperstability of a functional equation, Acta Math. Hungar., 2009, 124(1-2), 179-188

[21] Bahyrycz A., Piszczek M., Hyperstability of the Jensen functional equation, Acta Math. Hungar., 2014, 142(2), 353-365

[22] EL-Fassi Iz., Generalized hyerstability of a Drygas functional equation on a restricted domain using Brzdęk's fixed point theorem, J. Fixed Point Theory Appl., 2017, 19, 2529-2540

[23] Brzdęk J., Ciepliński K., Hyperstability and superstability, Abs. Appl. Anal., 2013(2013), Art. ID 401756

[24] Piszczek M., Remark on hyperstability of the general linear equation, Aequationes Math., 2014, 88(1-2), 163-168

[25] Brzdęk J., Chudziak J., Páles Z., A fixed point approach to stability of functional equations, Nonlinear Anal., 2011, 74(11), 6728-6732 